\title{
Preservice teacher disengagement with computer-based learning environments
}

\author{
Eric G. Poitras ${ }^{\mathrm{a}}$, Shan $\mathrm{Li}^{\mathrm{b}}$, Laurel Udy ${ }^{\mathrm{c}}$, Lingyun Huang ${ }^{\mathrm{d}}$, Susanne P. Lajoie ${ }^{\mathrm{e}}$ \\ ${ }^{a}$ University of Utah, US, eric.poitras@utah.edu, https://orcid.org/0000-0003-0563-7978 \\ ${ }^{b}$ McGill University, CA, shan.li2@mail.mcgill.ca, https://orcid.org/0000-0001-6001-1586 \\ ${ }^{c}$ University of Utah, US, laurel.udy@utah.edu, https://orcid.org/0000-0003-1218-8111 \\ ${ }^{d}$ McGill University, CA, francohuang168@gmail.com, https://orcid.org/0000-0002-7336-7079 \\ ${ }^{e}$ McGill University, CA, susanne.lajoie@mcgill.ca, https://orcid.org/0000-0003-2814-3962
}

\begin{abstract}
Investigating disengagement is a continuing concern within computer-based learning environments. Drawing upon several strands of research into preservice teacher learning with network-based tutors, this paper outlines an object orientation to conceptualize a type of disengaged behaviour referred to as carelessness. We further differentiate this construct in terms of carelessness towards one's own learning as opposed to other's learning. In support of our claims, we review research into carelessness in the context of nBrowser, an intelligent web browser designed to support preservice teachers learn about the pedagogical affordances of novel technologies while designing lesson plans. The key aspects of this research can be listed as follows: (1) a knowledge engineering approach to implement a set of production rules within the learning environment to detect instances of carelessness and intervene; (2) a data-driven approach to infer learner behaviours in their absence due to carelessness; and (3) a model-driven approach to improve the functioning of the learning environment despite instances of carelessness. We discuss the limitations of these different approaches and draw implications for future research into preservice teacher disengagement with computerbased learning environments.
\end{abstract}

Keywords: Learner disengagement; Carelessness; Computer-based learning environment; Network-based tutor

\section{Introduction}

Disengagement in the context of preservice teacher learning may be defined as a state that consists of three components (Fredricks, Blumenfeld, \& Paris, 2004): (a) behavioural (i.e. student actions and participation), (b) affective (i.e. positive and negative emotional reactions), and (c) cognitive (i.e. effortful information processing and thoughtfulness). As such, it is a multi-faceted construct that has been examined through different theoretical lenses and operationally defined in models of motivation, self-regulated learning, and cognitive disequilibrium as a dynamic state that fluctuates during complex learning (Azevedo, Moos, Johnson, \& Chauncey, 2010; D’Mello \& Graesser, 2011; Pekrun \& Linnenbrink-Garcia, 2014). Baker and Rossi (2013) argued that broad categories of disengaged behaviours in the context of computer-based learning environments (CBLEs) may include but are not limited to off-task behaviours, gaming the system, Without Thinking Fastidiously (WTF) behaviours, and careless behaviours within a learning environment. For example, learners who engage in the aforementioned behaviours are completely disengaged from the learning environment and task performance. Disengaged behaviours may include unrelated conversations, solitary activities, or inactivity. These typically manifest themselves in the context of interactions with CBLEs as extremely slow or fast actions, making frequent errors while writing strings of text, repeated and sequential errors, and errors made despite learners having shown proficiency in requisite skills (Baker, 2007). In contrast, learners who engage in gaming the system typically exploit properties of the system interface as a means to perform better, rather than to learn the material (e.g. quickly selecting answers until the correct one is found). The WTF category also comprises behaviours disconnected from the learning task, indicating that learners are not closely attending to the content, choosing instead to attend to superfluous details (e.g. running a similar experiment in a repeated manner by toggling settings back and forth (e.g. Rowe, McQuiggan, Robison, \& Lester, 2009; Wixon, Baker, Gobert, Ocumpaugh, \& Bachmann, 2012). Careless behaviours often occur when learners make errors, despite having shown to be proficient on 


\section{Preservice teacher disengagement with computer-based learning environments \\ Poitrasa, Lib, Udyc, Huangd, Lajoiee}

the task (e.g. a learner has made an error, despite the system indicating a high probability that the answer should have been correct given past performance).

In this paper, we propose a novel construct for behavioural disengagement, distinguishing carelessness towards one's own learning as opposed to others' learning. This object orientation added to the conceptualization of careless behaviours is warranted given our past research findings in preservice teacher learning with CBLEs. Preservice teachers engage in careless behaviours when they fail to rate the usefulness of information gained from an open educational resource. In doing so, teachers are careless towards others as the CBLE relies on ratings to suggest online resources to future preservice teachers while learning about the pedagogical affordances of technology and designing lesson plans. The next section reviews the research literature on learner disengagement and the methods used to address this issue.

\section{Learner Disengagement with Computer-Based Learning Environments}

Past research has shown that disengaged behaviours are often associated with poor learning when accounting for student performance on tasks (Aleven, McLaren, Roll, \& Koedinger, 2006; Baker, Corbett, \& Koedinger, 2004; Beck, 2005). Off-task behaviours may directly impact learning over time by decreasing opportunities available for students to practice skills (Cocea, Hershkovitz, \& Baker, 2009). In contrast, gaming the system has been shown to negatively impact learning over shorter and longer time durations (Rowe et al., 2009; Sabourin, Rowe, Mott, \& Lester, 2011). There have been several explanations offered for the onset of disengaged behaviours.

Student disengagement, such as gaming, is more likely to occur given the design of the learning environment, for instance when hints are overly abstract or ineffective, icons are unclear, or the problem under examination is uninteresting (Baker, 2007b). Student characteristics may also be predictive of the onset of such behaviours and have been shown to be more prominent among male students from urban schools than their female, rural, and suburban peers (Baker \& Gowda, 2010; Rowe et al., 2009). Students' motivational beliefs and affective reactions are important factors to consider as well, as research has shown that off-task and gaming behaviours are associated with disliking a topic, disliking the learning environment, believing in innate ability, believing that the tutor was not helpful, or lacking goal setting activities (Baker, 2007a; Walonoski \& Heffernan, 2006; Hershkovitz, Baker, Gobert, Wixon, \& Sao Pedro, 2013). Furthermore, disengaged behaviours are often followed by students reporting feeling either bored or frustrated; however, frustration is a much more adaptive reaction resulting in sustained effort on the task (Sabourin et al., 2011).

To address this issue, CBLEs may rely on virtual pedagogical agents who react negatively to instances when students behave in this manner and provide additional exercises in response. This has been shown to improve student learning and reduce further instances of disengaged behaviours (Baker et al., 2006). An alternative approach would be to provide students a visualization of past behaviours and explicit instruction on the broader impacts of disengagement towards learning, which has also been found to be helpful (Arroyo et al., 2007). In the following section, we examine further findings obtained from our own research into preservice teacher disengagement with nBrowser, a network-based tutor designed to support them in designing technology-infused lessons.

\section{Preservice Teacher Disengagement with nBrowser}

Network-based tutoring systems are similar to open-ended learning environments, allowing students to formulate their own goals and plans, select sources of information, and rely on scaffolds assimilate that information into task solutions (Hannafin 1995; Hill \& Land 1998). Network-based tutors rely on semantic web, content mining, and natural language processing techniques to represent information from online resources as nodes interrelated through links weighted on the basis of different semantic relations (Author(s), 2017; Author(s), 2018). In doing so, students are supported by the tutoring system in addressing issues stemming from disorientation and mental load intrinsic to learning about complex topics from hypermedia (Land, 2000; Author(s), 2006). The development of network-based tutors involves the use of web and text mining techniques to analyse the hypermedia content that characterize the knowledge and skills that students may acquire. Through analysing the properties of hypermedia elements, which are represented as an interrelated network of nodes, these systems reason and make instructional decisions.

As an example, the nBrowser system is an intelligent web browser designed to support preservice teachers in regulating their learning and gaining better understanding of the pedagogical affordances of digital technologies. The nBrowser system allows teachers to learn while performing two different types of tasks. In the create mode, preservice teachers create a lesson design on a topic of their choice and in the solve mode, they design a lesson to resolve an issue, such as the one described in the case of Linda the math teacher (see Figure 1). Preservice teachers navigate the web and design a lesson with the help of tools in the system, namely: (a) the lesson plan details panel where teachers define their objectives; (b) the lesson plan assets panel that allows them to search for useful information; and (c) the lesson plan builder panel, which allows them to edit a lesson. The recommender system featured in the lesson plan assets panel displays a description of an online resource, allowing preservice teachers to decide whether to navigate to the site. Furthermore, learners may choose to report a rating for the usefulness of an online resource using a five-point Likert scale slider bar ranging from least to most useful in designing a lesson. Finally, learners can review help in the form of hints that describe and exemplify structural elements of a lesson design as well as example lesson plan designs created 


\section{Preservice teacher disengagement with computer-based learning environments \\ Poitrasa, Lib, Udyc, Huangd, Lajoiee}

by preservice teachers with similar navigational profiles. Network-based tutors, such as nBrowser, are made of several system components that are common to ITS, including a domain model, learner model, instructional model, and user interface (see Shute \& Zapata-Rivera, 2012; Sottilare, Graesser, Hu, \& Holden, 2013).

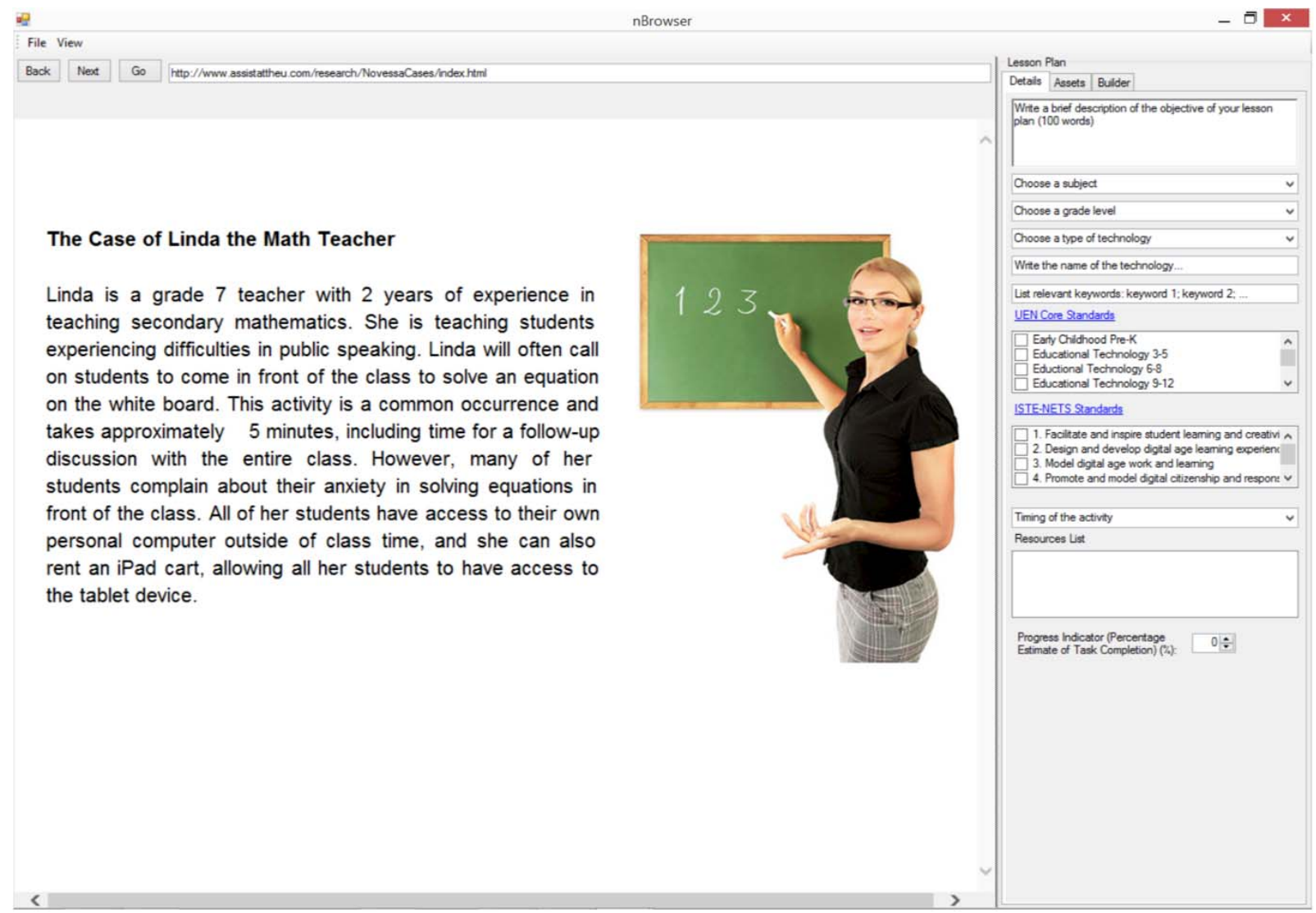

Fig. 1 The nBrowser main user interface (version 1.0)

The domain model of a network-based tutor consists of nodes that represent hypermedia elements (e.g. paragraphs, headers, and hyperlinks) interrelated by links weighted on the basis of different semantic relations. The underlying computational representation of the hypermedia content is developed using both web crawling, text pre-processing, and clustering techniques (i.e. automated methods), as well as human raters who rely on authoring tools such as the nCrawler software (i.e. manual methods). The resulting linguistic features that characterize hypermedia elements may be examined and compared in terms of different lexical, syntactic, cohesion, rhetorical, and reading ease indices to weight links spanning through nodes featured in the network (see McNamara, Crossley, \& Roscoe, 2013). As an example, a network-based tutor such as nBrowser makes instructional decisions on the basis of networks that represent collections of online resources, including the subject matter taught in the classroom, pedagogical practices and strategies, as well as educational technologies. The content of these online resources can be represented as nodes within the network at different hierarchical levels (e.g. from a single paragraph to all the elements featured in the page). The interrelationships among these nodes can be further characterized through the use of lexical overlap indices. In particular, the cosine similarity between sets of vectors of term mentions in each node, resulting in a weight value that ranges from 0 (i.e. terms mentioned in each node are different) to 1 (i.e. terms mentioned in each node are similar). The nBrowser system allows preservice teachers to navigate through nodes featured in the network while using tools to acquire and assimilate the relevant information during task performance.

At the same time, the learner model of nBrowser enables the tutoring system to continually update properties of the network on the basis of its underlying dimensions, user interactions with the system interface, and navigational trajectories. These properties are grounded within an information processing model of self-regulated learning to monitor students' learning progress, guide students' navigational choices, and provide the most suitable instructional content. As an example, the nBrowser system was designed to support preservice teachers' information seeking and acquisition while navigating the web to design a lesson plan that implements technologies in the classroom. The tutoring system tracks successful information-seeking behaviours by detecting the ratings provided by preservice teachers for the usefulness of online resources towards learning and task performance. Based on the self-report ratings, the domain model enables nBrowser to imitate how a human tutor would infer whether other sources of information are likely to be relevant given the learners' interests in a particular topic. The system relies on a spreading activation algorithm to 


\section{Preservice teacher disengagement with computer-based learning environments \\ Poitrasa, Lib, Udyc, Huangd, Lajoiee}

update the link weights while accounting for preservice teachers' ratings of online resource usefulness and whether another document mentions similar or different topics. As such, the topic similarity dimension of the network serves as the basis for the system to infer the utility of online resources to support preservice teachers' information seeking and acquisition.

Network-based tutoring systems rely on an instructional module that features amongst several types of scaffolds, a recommender system that analyses network properties and make decisions regarding the most suitable content to enhance learning. In the previously mentioned example, the topic similarity dimension of the network allows the system to continually update the link weights on the basis of the inferred utility of each online resource featured in the network. The nBrowser system relies on a recommender system to suggest online resources where the links spanning through the corresponding nodes are weighed the heaviest upon receiving a search request from preservice teachers. The system analyses each node and delivers the information to teachers in the form of a description and URL address, allowing teachers to navigate to the resource by clicking a button. At the same time, each recommendation made by the system is unique, as it tracks previous suggestions made to the learner.

The convergence effect was introduced by Poitras and Fazeli (2016) to refer to learning gains when navigation throughout the network of online resources is supported by nBrowser, that is, recommended successfully in terms of the utility of the underlying topic. According to the hypothesized effect, online resources are progressively weighted more heavily over time as the system infers the utility of not only specific nodes within the network but also other nodes that mention terms in common. In contrast, nodes that are inferred by the system to be less useful are weighted less heavily within the network. The rate at which a network is hypothesized to converge towards an optimal arrangement (i.e. the most useful online resources) and facilitate navigation towards these resources is expected to (1) increase as a function of the number of learner behaviours that indicate the utility of online resources and (2) decrease according to the size of the network or the number of nodes. If a larger number of learners rate the usefulness of online resources and the network contains a smaller number of nodes, then the network is expected to converge faster towards an optimal arrangement.

However, past research has shown that learners often fail to rate the usefulness of online resources recommended by nBrowser. Poitras, Fazeli, and Mayne (2018) found that the average number of site navigations logged in nBrowser was three views for each node in the network of online resources (M 3.11, Md 3.00, SD 2.38, Range: 0-10). The findings suggested that the preservice teachers viewed a majority of the sites featured in nBrowser, enabling them to rate $89 \%$ of online resources in terms of their usefulness for learning and task performance. Preservice teachers rated only $57 \%$ of nodes despite having viewed $89 \%$ of them in the browser window, which enabled them to submit ratings. The count of usefulness ratings is also heavily skewed; about one-third of the nodes was rated by the preservice teachers on a single occasion (36\%), and a tenth of the nodes was rated on two or three occasions (11\% and $8 \%$, respectively). Preservice teachers' carelessness in rating online resources has significant impacts towards the quality of suggestions made in nBrowser. In the following section, we review several methods to detect, intervene, address, and mitigate the problem, including knowledge engineered models as well as data-driven and model-driven approaches (see Figure 2).

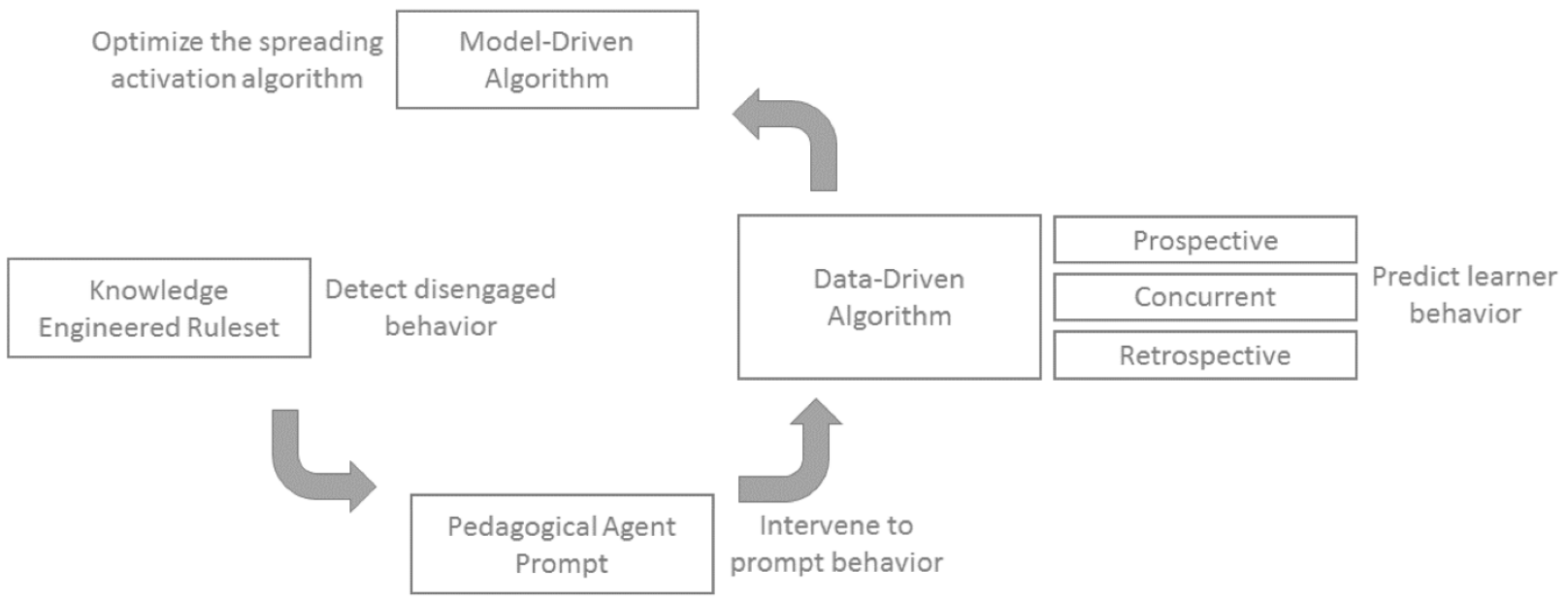

Fig. 2 Methods to Address Preservice Teacher Disengagement in nBrowser

\subsection{Knowledge Engineering Methods}

Knowledge engineered detectors of student disengagement rely on rational analysis by the researchers to define models and their parameters. Past research has implemented such models as production rules that are progressively refined and validated against student learning outcomes (Beal, Qu, \& Lee, 2006; Johns \& Woolf, 2006; Gong, Beck, \& Heffernan, 2010; Muldner, Burleson, Van de Sande, \& VanLehn, 2011). For instance, gaming behaviours may be 
defined by a set of rules, such as clicking through hints too rapidly to read the initial one delivered by the system or taking less time than some threshold value to respond (see Aleven, McLaren, Roll, \& Koedinger, 2004; Aleven et al., 2006). This approach is well-suited towards modelling carelessness towards others in the context of the nBrowser system, where the user interface is well structured and is amenable to valid assessments. Consider the following set of production rules, wherein:

(Step 1) IF a learner has repeated opportunities submit a rating of an online resource featured in the system database, (greater than 3 subsequent opportunities in a row) AND

(Step 2) IF a learner has failed to submit ratings on each opportunity ( 0 of 3 rating submission events recorded by the system),

(Step 3) THEN the learner is classified as being careless towards others.

This three-strike rule, albeit simplistic, would be considered a reasonable assumption to make in the context of the system, where learners had sufficient opportunity to engage in the target behaviour but repeatedly failed to do so. This set of rules would allow the nBrowser system to remind preservice teachers to submit usefulness ratings for online resources in an unobtrusive manner.

\subsection{Data-Driven Methods}

One such approach to the convergence problem is to rely on data-driven algorithms, where automated detectors of student disengagement are part of predictive models trained and evaluated on the basis of log trace data of user interactions. We distinguish between three types of models from the timing of predictions: prospective (before learning has taken place), concurrent (during learning), and retrospective models of student evaluations (after learning has occurred). Our past research has developed both concurrent and retrospective models of student behaviours as a means to address instances of carelessness towards others and improve upon the system capabilities to individualize instruction.

First, a C4.5 decision tree model was trained to detect useful and not useful ratings of information obtained from online resources on the basis of mouse cursor movement, position, or the combination of both, which is gathered throughout the course of learning with nBrowser (Author(s), 2018). To handle a continuously generated data stream or a sequence of time-stamped student interactions that are logged continuously at consecutive time points, time windows are commonly used to extract features. This approach is most effective in handling large volumes of data continuously generated in short periods of time while avoiding repeated analysis of the entire logs over individual student sessions to optimize memory usage. The log trace data for mouse cursor positions was set to refresh at approximately 10 coordinates per second; thus, we set the window size or forecasting horizon to 10 seconds, with increments of 2 seconds to extract features. In doing so, the window extracted six consecutive mouse cursor coordinate values (i.e. $0,2,4,6,8$, and 10 seconds) and five distance calculations from one coordinate to the next per window. We set the number of increments between successive window movements to 1 second, or 10 refresh events in the log trace data. In doing so, the first window contains observed mouse cursor coordinates and movements for period 1 through $t$, whereas the second window contains observed mouse cursor coordinates and movements for period 2 through $\mathrm{t} 1$, and so on. The resulting training set included 3,516 examples, after excluding examples prior to the rating submission event logged by the system using a cut-off point of 60 seconds. This was done given the need to predict ratings when learners failed to submit them. In these instances, learners neither used the slider bar nor selected the submit button. The best fitting model was found by optimizing pre- and post-pruning parameters and using feature discretization to distinguish positive and negative evaluations of online resources, with an average accuracy of $76.3 \%$ using 10 -fold stratified crossvalidation as the partitioning scheme.

Second, a RIPPER rule-induction algorithm was used to develop an automated detector to identify when learners navigated beyond an online resource without having submitted a rating (Author(s), 2018). An exhaustive search for optimal model parameters was conducted to fit the best performing set of rules. In particular, the sample ratio of the training set used to fit the model was set to $80 \%$ and $90 \%$ of examples to compare the outcomes. We varied the minimum amount of benefit obtained in relation to the information gain metric to prune the rule antecedents from $15 \%$ to $35 \%$, in increments of $2 \%$. The minimal ratio within a set of rules for the coverage of the most common class attribute value was set to vary between $80 \%$ and $100 \%$ in $2 \%$ increments. Finally, a combination of range values was evaluated, ranging from 2 to 20, to define rule antecedents of varying but equal ranges. The best fitting model was shown to classify positive and negative rating examples in a 10 -fold stratified cross-validation with $69 \%$ average predictive accuracy across all folds.

\subsection{Model-Driven Methods}

Our past research has relied on simulated learner experiments using nSimulator to model the manner in which the system suggests online resources to preservice teachers under controlled conditions. Simulated learners were introduced by VanLehn, Ohlsson, and Nason (1994), who recognized that "a technology that can simulate students presents an 


\section{Preservice teacher disengagement with computer-based learning environments}

Poitrasa, Lib, Udyc, Huangd, Lajoiee

opportunity for education" (p. 136). Moreover, simulated learners can be "designed to reflect the population of intended learners and scaled up to provide as large a sample size as desired" (Greer \& Mark, 2015, p. 389-390). Given the past probabilities of preservice teachers to navigate resources and rate their usefulness, computer simulations model the rate in which a specific resource is recommended by the system compared to distractor resources as a means to study the impact of carelessness towards others. In doing so, the nSimulator serves as a research platform for evaluating alternative algorithms under controlled conditions to enable a network to converge to an optimal state. The underlying assumption is that learners' carelessness towards others in failing to submit ratings can be mitigated through the design of efficient algorithms that allow networks to converge more quickly despite the lack of requisite learner interactions.

For this reason, nSimulator can serve as an intermediary step in an iterative cycle of improvement. First, nSimulator is used to ascertain the impact of authoring choices made in building network-based domain models by manipulating the instructional conditions and measuring the learning effects in terms of the quantity and quality of nodes visited, weight schemes, dimensions, and other properties. Because the authoring of the network-based tutor determines the range of instructional decisions made by the tutor, choices made about these factors are not trivial. For example, the choice of online resources to feature in the system database may constrain the performance of the recommender system. If too many resources are included in the database, it may take longer for the tutor to direct learners to the most useful ones. Second, nSimulator allows for the evaluation of algorithms that underlie the adaptive instructional features implemented in network-based tutors by examining how these operate in relation to a set of simulated learner behaviours modelled after actual learner behaviours. As an example, the spreading activation algorithm consists of a sigmoid activation function and underlies the convergence process once a rating of the usefulness of information gained from an online resource is detected by the system.

In conducting a series of simulations, we predicted that as the amount of online resources suggested by nBrowser increases, the system requires a greater amount of ratings to be submitted by learners to make effective recommendations (Poitras, Mayne, Huang, Doleck, Udy, \& Lajoie, 2018). The findings show that the system is more likely to recommend the target online resource under certain conditions, namely (a) smaller amount of resources (i.e. 10 nodes), as the odds are not significantly higher but remain relatively high and become increasingly variable across iterations comprising larger amounts of resources (i.e. 50-100 nodes); and (b) moderate numbers of simulated learners (i.e. 50-100 learners) where the system is more precise and sensitive in its recommendations than those obtained from larger samples (i.e. 200-500 learners). The findings build on previous methods by replicating each simulation to establish confidence intervals for each metric of the recommender system, improving upon previous findings where approximately 30 learners were necessary to optimize system recommendations from a small amount of online resources (Poitras \& Fazeli, 2017). Future research will examine alternate parameter values for the spreading activation algorithm under ideal conditions to ascertain the gains in efficiency for the rate of network convergence.

\section{Conclusions}

In summary, we have reviewed past research on learner behavioural disengagement related to preservice teachers in the context of CBLEs. In doing so, we argued in favour of an object orientation to conceptualize learner disengagement in terms of how carelessness may be directed towards either one's own learning or that of others. In support of this claim, we have examined the prevalence of carelessness in preservice teachers, which often fail to evaluate the usefulness of online resources recommended by nBrowser to other teachers. We outline several methods to address this issue. First, a knowledge engineered detector of carelessness that implemented in the system, allows nBrowser to prompt preservice teachers to report ratings. Second, a data-driven approach, where the system infers the usefulness of online resources when teachers fail to report them. Third, model-driven approaches that enable researchers to gain insights into the impact of carelessness towards system recommendations, resulting in changes to the recommender system parameters and algorithms. We call on the broader research community to take into consideration the role of carelessness in how preservice teachers design lesson plans in CBLEs as well as the proposed solutions outlined in this paper.

\section{References}

Aleven, V., McLaren, B. M., Roll, I. \& Koedinger, K. R. (2004). Toward tutoring help seeking: Applying cognitive modeling to meta-cognitive skills. In Proceedings of the 7th International Conference on Intelligent Tutoring Systems (pp. 227-239).

Aleven, V., McLaren, B., Roll, I. \& Koedinger, K. (2006). Toward meta-cognitive tutoring: A model of help seeking with a Cognitive Tutor. International Journal of Artificial Intelligence in Education, 16, 101-130.

Arroyo, I., Ferguson, K., Johns, J., Dragon, T., Meheranian, H., Fisher, D., Barto, A., Mahadevan, S. \& Woolf. B. P. (2007). Repairing disengagement with non-invasive interventions. In Proceedings of the 13th International Conference on Artificial Intelligence in Education (pp. 195-202). Springer, Berlin, Heidelberg. 
Azevedo, R., Moos, D. C., Johnson, A. M., \& Chauncey, A. D. (2010). Measuring cognitive and metacognitive regulatory processes during hypermedia learning: Issues and challenges. Educational Psychologist, 45(4), $210-223$. http://dx.doi.org/10.1080/00461520.2010.515934

Baker, R. S.J.d., \& Gowda, S. M. (2010). An analysis of the differences in the frequency of students' disengagement in urban, rural, and suburban high schools. In Proceedings of the 3rd International Conference on Educational Data Mining (pp. 11-20).

Baker, R. S.J.d. (2007a). Modeling and understanding students' off-task behavior in intelligent tutoring systems. In Proceedings of ACM CHI 2007: Computer-Human Interaction (pp. 1059-1068).

Baker, R. S.J.d. (2007b). Is gaming the system state-or-trait? Educational data mining through the multi-contextual application of a validated behavioral model. In Complete On-Line Proceedings of the Workshop on Data Mining for User Modeling at the 11th International Conference on User Modeling 2007 (pp. 76-80).

Baker, R. S.J.d., Corbett, A. T., Koedinger, K. R., Evenson, S. E., Roll, I., Wagner, A. Z., Naim, M., Raspat, J., Baker, D. J. \& Beck, J. (2006). Adapting to when students game an intelligent tutoring system. In Proceedings of the 8th International Conference on Intelligent Tutoring Systems (pp. 392-401).

Baker, R. S.J.d, Corbett, A. T., Koedinger, K. R. \& Wagner, A. Z. (2004). Off-task behavior in the Cognitive Tutor classroom: When students "game the system". In Proceedings of ACM CHI 2004: Computer-Human Interaction (pp. 383-390).

Baker, R. S.J.d., Rossi, L.M. (2013) Assessing the Disengaged Behaviors of Learners. In Sottilare, R., Graesser, A., Hu, X., \& Holden, H. (Eds.) Design Recommendations for Adaptive Intelligent Tutoring Systems - Volume 1 - Learner Modeling. U.S. Army Research Lab, Orlando, FL, pp. 155-166, 2013.

Beal, C. R., Qu, L. \& Lee, H. (2006). Classifying learner engagement through integration of multiple data sources. In Proceedings of the 21st National Conference on Artificial Intelligence (pp. 2-8). AAAI (American Association for Artificial Intelligence) Press.

Beck, J. (2005). Engagement tracing: using response times to model student disengagement. In Proceedings of the 12th International Conference on Artificial Intelligence in Education (pp. 88-95). Springer, Berlin, Heidelberg.

Buckley, B., Gobert, J. \& Horwitz, P. (2006). Using log files to track students' model-based inquiry. In Proceedings of the Seventh International Conference of the Learning Sciences (pp. 57-63). International Society of the Learning Sciences.

Cocea, M., Hershkovitz, A. \& Baker, R. S.J.d. (2009). The impact of off-task and gaming behaviors on learning: Immediate or aggregate? In Proceedings of the 14th International Conference on Artificial Intelligence in Education (pp. 507-514). Springer, Berlin, Heidelberg.

D’Mello, S., \& Graesser, A.C. (2011). The Half-Life of Cognitive-Affective States during Complex Learning. Cognition and Emotion, 25(7), 1299-1308.

Fredricks, J. A., Blumenfeld, P. C. \& Paris, A. H. (2004). School engagement: Potential of the concept, state of the evidence. Review of Educational Research, 74(1), 59-109.

Gong, Y., Beck, J. E. \& Heffernan, N. T. (2010). Comparing knowledge tracing and performance factor analysis by using multiple model fitting procedures. In Proceedings of the 10th International Conference on Intelligent Tutoring Systems (pp. 35-44).

Greer, J. \& Mark, M. (2015). Evaluation Methods for Intelligent Tutoring Systems Revisited. International Journal of Artificial Intelligence in Education, 389-390.

Hannafin, M. J. (1995). Open-ended learning environments: Foundations, assumptions, and implications for automated design. In R. Tennyson, A.E. Barron (Ed.), Automating Instructional Design: Computer-Based Development and Delivery Tools (pp. 101-129). New York: Springer-Verlag.

Hershkovitz, A., Baker, R. S.J.d., Gobert, J., Wixon, M., Sao Pedro, M. (2013). Discovery with Models: A Case Study on Carelessness in Computer-based Science Inquiry. American Behavioral Scientist, 57(10), 1480-1499.

Hill, J., \& Land, S. (1998). Open-ended learning environments: A theoretical framework and model for design. In M. Simonsen (Ed.), Proceedings of Selected Research and Development Presentations at the National Convention of the Association for Educational Communications and Technology. St Louis, MO: AECT. ERIC Document Reproduction Service No. ED423839.

Johns, J., \& Woolf, B. (2006). A dynamic mixture model to detect student motivation and proficiency. In Proceedings of the 21st National Conference on Artificial Intelligence (pp. 163-168). AAAI (American Association for Artificial Intelligence) Press.

Lajoie, S. P., \& Azevedo, R. (2006). Teaching and learning in technology-rich environments. In P. Alexander \& P. Winne (Eds.), Handbook of educational psychology (2nd ed.) (pp. 803-821). Mahwah, NJ: Erlbaum.

Land, S. M. (2000). Cognitive requirements for learning with open-ended learning environments. Educational Technology Research and Development, 48(3), 61-78.

McNamara, D. S., Crossley, S. A., \& Roscoe, R. (2013). Natural language processing in an intelligent writing strategy tutoring system. Behavior Research Methods, 45(2), 499-515. doi: 10.3758/s13428-012-0258-1.

Muldner, K., Burleson, W., Van de Sande, B. \& VanLehn, K. (2011). An analysis of students' gaming behaviors in an intelligent tutoring system: Predictors and impacts. User Modeling and User-Adapted Interaction, 21(1-2), 99-135. 


\section{Preservice teacher disengagement with computer-based learning environments \\ Poitrasa, Lib, Udyc, Huangd, Lajoiee}

Pekrun, R., \& Linnenbrink-Garcia, L. (2014). Introduction to emotions in education. In R. Pekrun \& L. LinnenbrinkGarcia (Eds.), Educational psychology handbook series. International handbook of emotions in education (pp. 110). New York, NY, US: Routledge/Taylor \& Francis Group.

Poitras, E. G., Doleck, T., \& Lajoie, S. P. (2018). Modeling student teachers' information seeking behaviors: Implications for adaptive scaffolding of lesson planning. Paper presented at the 2018 American Educational Research Association Annual Meeting. New York, NY.

Poitras, E. G., \& Fazeli, N. (2017). Simulating preservice teachers' information-seeking behaviors while learning with an intelligent web browser. Paper presented at the 2017 Society for Information Technology and Teacher Educational annual conference. Austin, TX.

Poitras, E., \& Fazeli, N. (2016). Mining the Edublogosphere to Enhance Teacher Professional Development. In Shalin Hai-Jew (Ed.), Social Media Data Extraction and Content Analysis. IGI Global.

Poitras, E., Doleck, T., Huang, L., Li, S., \& Lajoie, S. (2017). Advancing Teacher Technology Education Using OpenEnded Learning Environments as Research and Training Platforms. Australian Journal of Educational Technology, $33(3), 32-45$.

Poitras, E., Doleck, T., Huang, L., Li, S., \& Lajoie, S. (2018). nBrowser: An Intelligent Web Browser for Studying Self-Regulated Learning in Teachers' Use of Technology. In Robert Zheng (Ed.), Strategies for Deep Learning with Digital Technology: Theories and Practices in Education. Nova Science Publishers.

Poitras, E., Fazeli, N., \& Mayne, Z. (2018). Modeling Student Teachers' Information-Seeking Behaviors while Learning with Network-Based Tutors. Journal of Educational Technology \& Society.

Poitras, E., Mayne, Z., Huang, L., Doleck, T., Udy, L., \& Lajoie, S. (2018). Simulated student behaviors with intelligent tutoring systems: Applications for authoring and evaluating network-based tutors. In Scotty Craig (Ed.), Tutoring and Intelligent Tutoring Systems. Nova Publishers.

Rowe, J., McQuiggan, S., Robison, J. \& Lester, J. (2009). Off-task behavior in narrative-centered learning environments. In Proceedings of the 14th International Conference on Artificial Intelligence in Education (pp. 99106). Springer, Berlin, Heidelberg.

Sabourin, J., Rowe, J., Mott, B. \& Lester, J. (2011). When off-task is on-task: The affective role of off-task behavior in narrative-centered learning environments. In Proceedings of the 15th International Conference on Artificial Intelligence in Education (pp. 534-536). Springer, Berlin, Heidelberg.

Shute, V. J., \& Zapata-Rivera, D. (2012). Adaptive educational systems. In P. Durlach (Ed.), Adaptive technologies for training and education (pp. 7-27). New York, NY: Cambridge University Press.

Sottilare, R., Graesser, A., Hu, X., \& Holden, H. (Eds.). (2013). Design recommendations for Intelligent Tutoring Systems. Orlando, FL: U.S. Army Research Laboratory.

Vanlehn, K., Ohlsson, S., \& Nason, R. (1994). Applications of simulated students: An exploration. Journal of Artificial Intelligence in Education, 5(2), 135-175.

Walonoski, J. A. \& Heffernan, N. T. (2006). Prevention of off-task gaming behavior in intelligent tutoring systems. In Proceedings of the 8th International Conference on Intelligent Tutoring Systems (pp. 722-724). Springer-Verlag: Berlin.

Wixon, M., d Baker, R. S., Gobert, J. D., Ocumpaugh, J., \& Bachmann, M. (2012, July). WTF? detecting students who are conducting inquiry without thinking fastidiously. In Proceedings of the 20th International Conference on User Modeling, Adaptation, and Personalization (pp. 286-296). Springer, Berlin, Heidelberg. 